\title{
Observer-Based Robust Controller Design for Nonlinear Fractional-Order Uncertain Systems via LMI
}

\author{
Jing Qiu ${ }^{1}$ and Yude $\mathbf{J i}^{\mathbf{2}}$ \\ ${ }^{1}$ School of Information Sciences and Engineering, Hebei University of Science and Technology, Shijiazhuang, Hebei 050018, China \\ ${ }^{2}$ School of Sciences, Hebei University of Science and Technology, Shijiazhuang, Hebei 050018, China \\ Correspondence should be addressed to Yude Ji; ji_yude@163.com
}

Received 13 February 2017; Revised 4 June 2017; Accepted 17 July 2017; Published 28 August 2017

Academic Editor: Michele Betti

Copyright (c) 2017 Jing Qiu and Yude Ji. This is an open access article distributed under the Creative Commons Attribution License, which permits unrestricted use, distribution, and reproduction in any medium, provided the original work is properly cited.

\begin{abstract}
We discuss the observer-based robust controller design problem for a class of nonlinear fractional-order uncertain systems with admissible time-variant uncertainty in the case of the fractional-order satisfying $0<\alpha<1$. Based on direct Lyapunov approach, a sufficient condition for the robust asymptotic stability of the observer-based nonlinear fractional-order uncertain systems is presented. Employing Finsler's Lemma, the systematic robust stabilization design algorithm is then proposed in terms of linear matrix inequalities (LMIs). The efficiency and advantage of the proposed algorithm are finally illustrated by two numerical simulations.
\end{abstract}

\section{Introduction}

With the help of fractional-order derivatives, many engineering plants and processes can be described more adequately and accurately; fractional-order systems (FOS) have attracted more and more attentions from the application point of view $[1,2]$, such as heat conduction electronic and abnormal diffusion. The main reason for successful applications of fractional calculus is that these new fractional-order models are more accurate than integer-order models; that is, there are more degrees of freedom in the fractional-order models. In recent years, considerable attention has been paid to fractional control systems whose processes and/or controllers are fractional-order. Since fractional-order derivatives are nonlocal and have weakly singular kernels, the stability analysis of fractional-order differential equations is more complex than that of integer-order differential equations. In this sense, the stability of FOS has been extensively studied, for example, the continuous time FOS [3-5] and discrete time FOS [6]. In terms of linear matrix inequalities (LMIs), the stability condition has been given for a continuous FOS with fractional-order $0<\alpha<1$ in [7] and $1 \leq \alpha<2$ in [8]. Moreover, the Mittag-Leffler stability of nonlinear FOS was derived in $[9,10]$. In order to obtain the stability of nonlinear fractional-order time-varying systems, some authors [11, 12] have proposed the extension of Lyapunov stability theorem with fractional-order to prove the stability of FOS. However, using this technique is often a really hard task, because finding a Lyapunov candidate function is more complex in the case of fractional-order. Trigeassou and Maamri study the initial condition problem by the frequency-distribution state-space model in [13]. The finite-dimensional fractionalorder state-space model with the zero initial conditions was equivalent to an infinite-dimensional integer-order statespace model and two specific types of Lyapunov candidate functions were proposed to investigate the stability problem of fractional differential equations (FDEs) in [14]. The stability conditions of singular FOS and fractional-order delay system are given in [15-19]. Recently, [20,21] provides a good survey of the methods available to analyze the stability of fractional-order differential systems, the reader may refer to them and the references therein.

In many practical applications, due to technical or economic reasons, the states of considered system are not easily obtained and the output may be contaminated by the measurement noises $[22,23]$. In this case, the state estimators 
and observer-based control are often necessary. The approximate observability and pseudo state estimation of FOS are discussed in [24]. Some results of observer design for FOS and integer-order systems are studied in [25-28]. In addition, an observer for which the estimation error diverges by a small perturbation in the observer gain is referred to as fragile or nonresilient. The observability of FOS has been addressed in several papers. In the linear case, it is the well-known Luenberger's observer. Using matrix's singular value decomposition (SVD) and linear matrix inequality (LMI) techniques, the observer-based robust control for linear fractional-order uncertain systems with fractionalorder $1 \leq \alpha<2$ was investigated in [25]. A simple method to design a functional observer for linear FOS was presented in [29]. Output stabilisability and observer-based switched control design problems for systems that switch among a finite set of controllable and observable linear subsystems at any given switching frequency was studied in [30]. New simple LMIs are proposed to guarantee the stability of a class of linear fractional-order uncertain systems by means of a fractional-order deterministic observer in [31]. A number of contributions to the extension of the Luenberger's observer for nonlinear systems have been proposed in the literature (see [32, 33], and references therein). The observer-based control is usually applied when we do not have access to all the states of the system. The notion of detectability has been extended to nonlinear systems in [34]. Based on the principle of separation, the nonlinear observer-based control is designed in [35]. Reference [36] concerns observer-based robust control problem for a class of fractional-order complex dynamical networks. In [37], by using the properties of Mittag-Leffler function and the Gronwall-Bellman inequality, two sufficient conditions on the global asymptotic stability for a class of nonlinear FOS with the fractional-orders $0<\alpha<1$ and $1<\alpha<2$ are derived, respectively. In the formulation of LMIs, the sufficient conditions for asymptotical stability of the state error dynamics are derived from the fractional-order direct Lyapunov theorem with fractional-order belonging to $0<\alpha<1$ in [38]. A design method of nonfragile observerbased robust control for nonlinear fractional-order uncertain system has been presented in [39]. An adaptive nonfragile observer for Lipschitz nonlinear systems was designed by LMI technique in [40]. Recently, a nonfragile fractional-order nonlinear observer design for nonlinear FOS was derived via LMI in [26], and its main advantage reflected in the selection of the Lyapunov candidate function. Based on the continuous frequency distributed equivalent model and indirect Lyapunov approach, an LMI approach to design a full-order observer for nonlinear FOS has been presented in [41]. Attempts are made to design a reduced-order observer for a class of nonlinear Lipschitz FOS in [42]. The robust stabilization problem for nonlinear fractional-order uncertain systems with admissible time-variant uncertainty in system matrix and bounded perturbation on the observer gain have been investigated in [43]. The sliding mode observer for state and fault estimation for nonlinear FOS is designed in [44]. Note that most of the above-mentioned results concern the stability and stabilization of linear and nonlinear
FOS. The nonlinear nonfragile fractional-order observerbased stabilization problem for FOS is still open to our best knowledge.

Motivated by the above discussions, we propose sufficient condition for robust observer-based controller design of nonlinear fractional-order uncertain systems in this paper. A novel LMI design strategy is presented by using the direct Lyapunov approach; the systematic robust stabilization design algorithm is then proposed in terms of LMIs by employing Finsler's Lemma. It is worth noting that the proposed LMI condition is solved without any additional restrictive conditions, unlike $[25,39,43]$ as it bases on the matrix's SVD. We also consider uncertainties in the system matrices which have not been addressed in the previous literature for input matrix $B$.

Throughout this paper, $\mathbb{R}^{n}$ denotes the $n$-dimensional Euclidean space, $\mathbb{R}^{n \times m}$ is the set of all $n \times m$ real matrices, and $I$ and 0 stand for identity matrix and zero matrix with appropriate dimensions, respectively. $X>0(<0)$ indicates that the matrix $X$ is positive (negative) definite, $\operatorname{diag}\left\{a_{1}, a_{2}, \ldots, a_{n}\right\}$ denotes the block-diagonal matrix, and $\operatorname{Sym}\{M\}$ denotes the expression $M+M^{T}$. $X^{T}$ and $X^{-1}$ represent the transpose and the inverse of matrix $X$, respectively, and the symbol $*$ is used to denote the transposed elements in the symmetric positions of a matrix.

The paper is organized as follows. In Section 2, the preliminary results and problem formulation are presented. The main results are derived in Section 3. Numerical simulation examples that demonstrate the effectiveness of the method are shown in Section 4. Finally, Section 5 concludes with a summary of the obtained results.

\section{Preliminary Results and Problem Formulation}

There are several definitions of fractional-order derivative in [1]. The Riemann-Liouville and Caputo fractional-order derivative are most widely used. In this paper, we adopt the Riemann-Liouville fractional-order derivative definition.

Definition 1 (see [1]). The Riemann-Liouville type fractionalorder derivative of order $\alpha>0$ for a function $f: \mathbb{R}^{+} \rightarrow \mathbb{R}$ is defined by

$$
D^{\alpha} f(t)=\frac{1}{\Gamma(m-\alpha)} \frac{\mathrm{d}^{m}}{\mathrm{~d} t^{m}} \int_{0}^{t} \frac{f(s)}{(t-s)^{\alpha+1-m}} \mathrm{~d} s,
$$

where $\Gamma(\cdot)$ is the well-known Gamma function which is made up of $\Gamma(q)=\int_{0}^{\infty} t^{q-1} e^{-t} \mathrm{~d} t$ and $m$ is an integer satisfying $m-$ $1<\alpha \leq m$.

Remark 2. Although the problem of observer-based robust control for the fractional-order systems is researched in some references $[25,43]$ and $[26,39]$ are based on the Caputo type and Riemann-Liouville type fractional-order derivative, respectively, but only [39] considers the initialization. In order to satisfy the consistency for physical behavior of the system, we adopt the Riemann-Liouville fractional-order derivative definition in this paper. 
Remark 3. Although Caputo type definition of fractionalorder derivative is a modification of the Riemann-Liouville definition and has the advantage of dealing with initial value problems in a proper way, Caputo's definition does not permit taking into account initial conditions in a coherent way. Even if the Riemann-Liouville derivative reflects more precisely the typical long memory behavior of FOS, this derivative definition has a major limitation that the initial conditions are in terms of fractional-order derivatives of the variable. In contrast, the definition given by Caputo type derivative needs the initial conditions which are specified in the usual way.

Lemma 4 (see [45]). The FOS $D^{\alpha} y(t)=v(t)$ with $0<\alpha<$ $1, v(t) \in \mathbb{R}$, and $y(t) \in \mathbb{R}$ is a continuous linear frequency distributed system. Its frequency distributed state $z(\omega, t) \in \mathbb{R}$ which is also called the true state of FOS satisfies

$$
\frac{\partial z(\omega, t)}{\partial t}=-\omega z(\omega, t)+v(t)
$$

and the output $y(t)$ is the weighted integral

$$
y(t)=\int_{0}^{\infty} \mu_{\alpha}(\omega) z(\omega, t) \mathrm{d} \omega,
$$

with the frequency weighting function

$$
\mu_{\alpha}(\omega)=\frac{\sin (\alpha \pi)}{\omega^{\alpha} \pi}>0
$$

Remark 5. Lemma 4 implies the infinite dimension of fractional-order state. This Lemma derives from zero initial condition, while the system response by definition of Riemann-Liouville or Caputo corresponds to the response of frequency distributed model with specific initial value [46]. With the help of equivalent frequency distributed model, the stability could be analyzed via Lyapunov technique where true state replaces pseudo state [47]. The definition of the initial conditions of fractional differential systems is an old problem which has not received satisfactory solutions. Neither Riemann-Liouville nor Caputo's definitions for fractional differentiation can be used to take into account initial conditions in a convenient way from a physical point of view [48]. Owing to the frequency distributed model, and particularly with these true state variables, many applications can be considered, as observers, state-space controllers, and stability analysis by Lyapunov technique [47].

Remark 6. Recently, some references $[49,50]$ have shown that fractional models are physically inconsistent models and such a model can be seen as a diffusion equation on an infinite-dimensional space. As a consequence, if a real system is represented by a fractional model, some properties (e.g., observability, controllability) of the model do not necessarily reflect the properties of the real system.

Lemma 7 (see [51]). Given real matrices $M$ and $N$ with appropriate dimensions, $M F(t) N+N^{T} F^{T}(t) M^{T}<0$ for all
$F(t)$ satisfying $F^{T}(t) F(t) \leq I$ if and only if there exists a real scalar $\varepsilon>0$ such that $\varepsilon M M^{T}+(1 / \varepsilon) N^{T} N<0$.

Lemma 8 (Finsler's Lemma, see $[52,53]$ ). Let $\zeta \in \mathbb{R}^{n}, Q \in$ $\mathbb{R}^{n \times n}, B \in \mathbb{R}^{s \times n}$, and $\operatorname{rank}(B)<n$. Then

$$
\zeta^{T} Q \zeta<0, \quad \forall \zeta \neq 0 \text { such that } B \zeta=0
$$

holds if and only if any of the following conditions hold:

(a) $B_{\perp}^{T} Q B_{\perp}<0$, where $B_{\perp}$ is such that $B B_{\perp}=0$ and $B B^{T}+$ $B_{\perp}^{\bar{T}} B_{\perp}>0$

(b) $\exists \mu \in \mathbb{R}: Q-\mu B^{T} B<0$;

(c) $\exists V \in \mathbb{R}^{n \times s}: Q+V B+B^{T} V^{T}<0$. form:

Consider the nonlinear FOS described by the following

$$
\begin{aligned}
D^{\alpha} x(t)= & (A+\Delta A(t)) x(t)+(B+\Delta B(t)) u(t) \\
& +\Phi(x(t)) \\
y(t)= & C x(t)
\end{aligned}
$$

where $D^{\alpha}$ denotes the Riemann-Liouville type fractionalorder derivative, $0<\alpha<1$ is the fractional commensurate order, $x(t) \in \mathbb{R}^{n}, u(t) \in \mathbb{R}^{q}$, and $y(t) \in \mathbb{R}^{m}$ are the pseudo state, input and output vector at time $t$, respectively; $A \in$ $\mathbb{R}^{n \times n}, B \in \mathbb{R}^{n \times q}$, and $C \in \mathbb{R}^{m \times n}$ are system, input, and output matrix, respectively. $\Delta A(t)$ and $\Delta B(t)$ are time-variant matrices denoting the norm-bounded parameter uncertainties and are assumed to be of the following form:

$$
[\Delta A(t) \quad \Delta B(t)]=\left[\begin{array}{lll}
D_{A} F_{A}(t) E_{A} & D_{B} F_{B}(t) E_{B}
\end{array}\right]
$$

where $M_{A}, M_{B}$ and $N_{A}, N_{B}$ are known constant matrices with appropriate dimensions, and the uncertain matrices $F_{A}(t)$ and $F_{B}(t)$ satisfy

$$
\begin{aligned}
& F_{A}(t) F_{A}^{T}(t) \leq I, \\
& F_{B}(t) F_{B}^{T}(t) \leq I .
\end{aligned}
$$

Moreover, $\Phi(\cdot): \mathbb{R}^{n} \rightarrow \mathbb{R}^{n}$ is a nonlinear function which is Lipschitz in $x$ with Lipschitz constant $\gamma$ :

$$
\left\|\Phi\left(x_{1}\right)-\Phi\left(x_{2}\right)\right\| \leq \gamma\left\|x_{1}-x_{2}\right\|
$$

for all $x_{1}(t), x_{2}(t) \in \mathbb{R}^{n}$ and

$$
\Phi(0)=0
$$

We regard $t_{0}=0$ as the initial time. We suppose that the system was at stationary state at time $l(-\infty<l<0)$ and that the system was initialized from the time $l$ in the past. As 
mentioned in [45], a FOS requires complicated initialization. For FOS (6), the following initialization function is used:

$$
x_{0}=\lim _{t \rightarrow 0} \frac{1}{\Gamma(1-\alpha)} \int_{l}^{t}(t-\tau)^{-\alpha} x(\tau) \mathrm{d} \tau
$$

Without loss of generality, we also assume that FOS (6) is observable and controllable.

Assumption 9. The output matrix $C$ of system (6) is full row $\operatorname{rank}(\operatorname{rank}(C)=m)$, and, therefore, there exists nonsingular matrix $T$ such that

$$
C T=\left[\begin{array}{ll}
I & 0
\end{array}\right]
$$

Remark 10. For any given $C$, the corresponding $T$ is not unique in general. One solution for $T$ as discussed in [54] is

$$
T=\left[C^{T}\left(C C^{T}\right)^{-1} C^{\perp}\right]
$$

where $C^{\perp}$ is called an orthogonal basis for the null space of $C$ satisfying $C C^{T}=0$.

In this paper, the purpose is to study the asymptotical stabilization of nonlinear FOS (6) with a Luenberger-type fractional-order nonfragile observer

$$
\begin{aligned}
D^{\alpha} \widehat{x}(t)= & \widehat{A} \hat{x}(t)+B u(t)+\Phi(\widehat{x}(t)) \\
& +(L+\Delta L(t))(y(t)-\widehat{y}(t)), \\
\widehat{y}(t)= & C \widehat{x}(t),
\end{aligned}
$$

with the pseudo state estimated feedback controller:

$$
u(t)=K \widehat{x}(t)
$$

where

$$
\Delta L(t)=D_{L} F_{L}(t) E_{L}
$$

in which $D_{L}$ and $E_{L}$ are known constant matrices, and $F_{L}(t)$ is unknown matrices with Lebesgue measurable elements satisfying

$$
F_{L}(t) F_{L}^{T}(t) \leq I
$$

$\widehat{x}(t) \in \mathbb{R}^{n}$ is the estimate of system pseudo state $x(t), K \in$ $\mathbb{R}^{q \times n}$ is the systems control gain matrix, and $\widehat{A} \in \mathbb{R}^{n \times n}, L \in$ $\mathbb{R}^{n \times m}$ are the systems observer gain matrices.

Let $e(t)=x(t)-\widehat{x}(t)$ denote the observation error of systems. Hence, under the controller (15), the closed-loop control FOS are obtained by

$$
\begin{aligned}
D^{\alpha} \widehat{x}(t)= & (\widehat{A}+B K) \widehat{x}(t)+(L+\Delta L(t)) C e(t) \\
& +\Phi(\widehat{x}(t))
\end{aligned}
$$

$$
\begin{aligned}
D^{\alpha} e(t)= & (A+\Delta A(t)-\widehat{A}+\Delta B(t) K) \widehat{x}(t) \\
& +(A+\Delta A(t)-(L+\Delta L(t)) C) e(t) \\
& +\Phi(x(t))-\Phi(\widehat{x}(t)),
\end{aligned}
$$

which is equivalent to

$$
D^{\alpha} X(t)=A_{\mathrm{cl}} X(t)+\Psi(X(t))
$$

where

$$
\begin{aligned}
& X(t)=\left[\begin{array}{l}
\hat{x}(t) \\
e(t)
\end{array}\right], \\
& \Psi(X(t))=\left[\begin{array}{c}
\Phi(\widehat{x}(t)) \\
\Phi(x(t))-\Phi(\widehat{x}(t))
\end{array}\right], \\
& A_{\mathrm{cl}} \\
& =\left[\begin{array}{cc}
\widehat{A}+B K & (L+\Delta L(t)) C \\
A+\Delta A(t)-\widehat{A}+\Delta B(t) K & A+\Delta A(t)-(L+\Delta L(t)) C
\end{array}\right] .
\end{aligned}
$$

Therefore, the design of observer-based controller problem can be converted into the robust asymptotical stability problem of nonlinear FOS (19).

Remark 11. On the one hand, utilizing a simple modification and the same discussions in [24], we can get that the real state of nonlinear FOS (6) is not completely observable but approximately observable assuming that the integer-order subsystem state is observable. On the other hand, as pointed out in [24], in many applications, estimating the real state is not useful and only an estimation of the variable $x(t)$ as state-space representation (6) is enough. Therefore, we make the fractional-order Luenberger like observer be expressed as (14).

Remark 12. Different from the existing Luenberger-type observer (see, e.g., [26]), nonlinear fractional-order observer (14) contains two unknown system matrices $\widehat{A}$ and $L$, which give a chance in order to better adjust the dynamical behaviors of observer-based FOS.

\section{Main Results}

In this section a new method for observer-based controller design for nonlinear FOS (6) is proposed based on a common quadratic Lyapunov function; we obtain the following results.

Theorem 13. Given positive scalar design parameter $\lambda$, the observer-based closed-loop system (19) is robustly asymptotically stable if there exist symmetric positive definite matrices $\mathscr{P}_{1}, \mathscr{P}_{2} \in \mathbb{R}^{n \times n}$, full rank matrix $Q_{11} \in \mathbb{R}^{m \times m}$, matrices $Q_{21} \in \mathbb{R}^{(n-m) \times m}, Q_{22} \in \mathbb{R}^{(n-m) \times(n-m)}, \mathscr{A} \in \mathbb{R}^{n \times n}, \mathscr{K} \in \mathbb{R}^{q \times n}$, 
and $\mathscr{L} \in \mathbb{R}^{n \times m}$, and two real scalars $\varepsilon_{1}>0, \varepsilon_{2}>0$ such that the following LMI condition holds:

$$
\left[\begin{array}{cccccccc}
\Theta_{11} & \Theta_{12} & \mathscr{P}_{1} & 0 & \mathscr{P}_{1} E_{A}^{T} & \mathscr{K}^{T} E_{B}^{T} & 0 & \lambda \widehat{Q} \\
* & \Theta_{22} & 0 & \mathscr{P}_{2} & \mathscr{P}_{2} E_{A}^{T} & 0 & \mathscr{P}_{2} C^{T} E_{L}^{T} & \mathscr{P}_{2}-Q^{T} T^{T}-\lambda \widehat{Q} \\
* & * & -\frac{\varepsilon_{1}}{\gamma^{2}} I & 0 & 0 & 0 & 0 & 0 \\
* & * & * & -\frac{\varepsilon_{1}}{\gamma^{2}} I & 0 & 0 & 0 & 0 \\
* & * & * & * & -\varepsilon_{2} I & 0 & 0 & 0 \\
* & * & * & * & * & -\varepsilon_{2} I & 0 & 0 \\
* & * & * & * & * & * & -\varepsilon_{2} I & 0 \\
* & * & * & * & * & * & 0 & -\lambda\left(T Q+Q^{T} T^{T}\right)
\end{array}\right]<0
$$

where

$$
\begin{aligned}
\Theta_{11}= & \operatorname{Sym}\{\mathscr{A}+B \mathscr{K}\}+\varepsilon_{1} I+\varepsilon_{2} D_{L} D_{L}^{T}, \\
\Theta_{12}= & \mathscr{P}_{1} A^{T}-\mathscr{A}^{T}+\widehat{Q}-\varepsilon_{2} D_{L} D_{L}^{T}, \\
\Theta_{22}= & \operatorname{Sym}\left\{A \mathscr{P}_{2}-\widehat{Q}\right\}+\varepsilon_{1} I \\
& +\varepsilon_{2}\left(D_{A} D_{A}^{T}+D_{B} D_{B}^{T}+D_{L} D_{L}^{T}\right), \\
Q= & {\left[\begin{array}{ll}
Q_{11} & 0 \\
Q_{21} & Q_{22}
\end{array}\right] \in \mathbb{R}^{n \times n}, } \\
\widehat{Q}= & {\left[\begin{array}{ll}
\mathscr{L} & 0
\end{array}\right] \in \mathbb{R}^{n \times n}, }
\end{aligned}
$$

with matrix T satisfying (12). Moreover, the stabilizing control feedback and the observer gains in (14) and (15) are given by

$$
\begin{aligned}
\widehat{A} & =\mathscr{A} \mathscr{P}_{1}^{-1}, \\
K & =\mathscr{K} \mathscr{P}_{1}^{-1}, \\
L & =\mathscr{L} Q_{11}^{-1} .
\end{aligned}
$$

Proof. It follows from Lemma 4 that the closed-loop system (19) can be written as

$$
\begin{aligned}
\frac{\partial Z(\omega, t)}{\partial t} & =-\omega Z(\omega, t)+A_{\mathrm{cl}} X(t)+\Psi(X(t)), \\
X(t) & =\int_{0}^{\infty} \mu_{\alpha}(\omega) Z(\omega, t) \mathrm{d} \omega
\end{aligned}
$$

where $Z(\omega, t)=\left[Z_{1}(\omega, t), Z_{2}(\omega, t), \ldots, Z_{2 n}(\omega, t)\right]^{T}$. tion as

We define the following monochromatic Lyapunov func-

$$
V(t)=\int_{0}^{\infty} \mu_{\alpha}(\omega) Z(\omega, t)^{T} P Z(\omega, t) \mathrm{d} \omega
$$

where $P \in \mathbb{R}^{2 n \times 2 n}$ is a symmetric positive definite matrix.
Taking the derivative of (26), it then causes

$$
\begin{aligned}
& \frac{\mathrm{d} V(t)}{\mathrm{d} t}=\int_{0}^{\infty} \mu_{\alpha}(\omega) \\
& \cdot\left(-\omega Z(\omega, t)+A_{\mathrm{cl}} X(t)+\Psi(X(t))\right)^{T} \\
& \cdot P Z(\omega, t) \mathrm{d} \omega+\int_{0}^{\infty} \mu_{\alpha}(\omega) Z(\omega, t)^{T} \\
& \cdot P\left(-\omega Z(\omega, t)+A_{\mathrm{cl}} X(t)+\Psi(X(t))\right) \mathrm{d} \omega \\
& =-2 \int_{0}^{\infty} \mu_{\alpha}(\omega) \omega Z(\omega, t)^{T} P Z(\omega, t) \mathrm{d} \omega \\
& +X^{T}(t) \operatorname{Sym}\left\{P A_{\mathrm{cl}}\right\} X(t)+\Psi(X(t))^{T} P X(t) \\
& +X^{T}(t) P \Psi(X(t)) .
\end{aligned}
$$

According to the Lyapunov theory, the integral-order system (25) is robustly stable if $\mathrm{d} V(t) / \mathrm{d} t<0$, that is, if

$$
\begin{aligned}
& X^{T}(t) \operatorname{Sym}\left\{P A_{\mathrm{cl}}\right\} X(t)+\Psi(X(t))^{T} P X(t) \\
&+X^{T}(t) P \Psi(X(t))<0 .
\end{aligned}
$$

Using Lemma 7 on the second term of inequality (28) for any real scalar $\varepsilon_{1}>0$, it yields

$$
\begin{aligned}
\frac{\mathrm{d} V(t)}{\mathrm{d} t} \leq & X^{T}(t) \operatorname{Sym}\left\{P A_{\mathrm{cl}}\right\} X(t)+\varepsilon_{1} X^{T}(t) P P X(t) \\
& +\varepsilon_{1}^{-1} \Psi(X(t))^{T} \Psi(X(t)) .
\end{aligned}
$$

It implies from (9) and (10) that

$$
\begin{aligned}
& \Psi(X(t))^{T} \Psi(X(t)) \\
&= {\left[\begin{array}{ll}
\Phi^{T}(\widehat{x}(t)) & (\Phi(x(t))-\Phi(\widehat{x}(t)))^{T}
\end{array}\right]\left[\begin{array}{c}
\Phi(\widehat{x}(t)) \\
\Phi(x(t))-\Phi(\widehat{x}(t))
\end{array}\right] } \\
&= \Phi^{T}(\widehat{x}(t)) \Phi(\widehat{x}(t)) \\
&+(\Phi(x(t))-\Phi(\widehat{x}(t)))^{T}(\Phi(x(t))-\Phi(\widehat{x}(t))) \\
& \leq \gamma^{2} X^{T}(t) X(t) .
\end{aligned}
$$


Substituting (30) into inequality (29), it yields

$$
\begin{aligned}
\frac{\mathrm{d} V(t)}{\mathrm{d} t} \leq & X^{T}(t) \operatorname{Sym}\left\{P A_{\mathrm{cl}}\right\} X(t) \\
& +X^{T}(t)\left(\varepsilon_{1} P P+\varepsilon_{1}^{-1} \gamma^{2} I\right) X(t) .
\end{aligned}
$$

$$
\begin{aligned}
\operatorname{Sym}\left\{P A_{\mathrm{cl}}\right\} & =\operatorname{Sym}\left\{\left[\begin{array}{cc}
P_{1}(\widehat{A}+B K) & P_{1}(L+\Delta L(t)) C \\
P_{2}(A+\Delta A(t)-\widehat{A}+\Delta B(t) K) & P_{2}(A+\Delta A(t)-(L+\Delta L(t)) C)
\end{array}\right]\right\} \\
& =\operatorname{Sym}\left\{\left[\begin{array}{cc}
P_{1}(\widehat{A}+B K) & P_{1} L C \\
P_{2}(A-\widehat{A}) & P_{2}(A-L C)
\end{array}\right]\right\}+\operatorname{Sym}\left\{\left[\begin{array}{cc}
0 & P_{1} \Delta L(t) C \\
P_{2} \Delta A(t)+P_{2} \Delta B(t) K & P_{2} \Delta A(t)-P_{2} \Delta L(t) C
\end{array}\right]\right\}
\end{aligned}
$$

By developing $\Delta A(t), \Delta B(t)$, and $\Delta L(t)$, according to (7), (16), and applying Lemma 7 to inequality (33) for any real scalar $\varepsilon_{2}>0$, we have

$$
\begin{aligned}
& \operatorname{Sym}\left\{P A_{\mathrm{cl}}\right\}=\operatorname{Sym}\left\{\left[\begin{array}{cc}
P_{1}(\widehat{A}+B K) & P_{1} L C \\
P_{2}(A-\widehat{A}) & P_{2}(A-L C)
\end{array}\right]\right\} \\
& +\operatorname{Sym}\left\{\left[\begin{array}{cc}
0 & P_{1} D_{L} F_{L}(t) E_{L} C \\
P_{2} D_{A} F_{A}(t) E_{A}+P_{2} D_{B} F_{B}(t) E_{B} K & P_{2} D_{A} F_{A}(t) E_{A}-P_{2} D_{L} F_{L}(t) E_{L} C
\end{array}\right]\right\} \\
& =\operatorname{Sym}\left\{\left[\begin{array}{cc}
P_{1}(\widehat{A}+B K) & P_{1} L C \\
P_{2}(A-\widehat{A}) & P_{2}(A-L C)
\end{array}\right]\right\} \\
& +\operatorname{Sym}\left\{\left[\begin{array}{ccc}
0 & 0 & P_{1} D_{L} \\
P_{2} D_{A} & P_{2} D_{B} & -P_{2} D_{L}
\end{array}\right]\left[\begin{array}{lll}
F_{A}(t) & & \\
& F_{B}(t) & \\
& & F_{L}(t)
\end{array}\right]\left[\begin{array}{cc}
E_{A} & E_{A} \\
E_{B} K & 0 \\
0 & E_{L} C
\end{array}\right]\right\} \\
& \leq \operatorname{Sym}\left\{\left[\begin{array}{cc}
P_{1}(\widehat{A}+B K) & P_{1} L C \\
P_{2}(A-\widehat{A}) & P_{2}(A-L C)
\end{array}\right]\right\}+\varepsilon_{2}\left[\begin{array}{ccc}
0 & 0 & P_{1} D_{L} \\
P_{2} D_{A} & P_{2} D_{B} & -P_{2} D_{L}
\end{array}\right]\left[\begin{array}{ccc}
0 & 0 & P_{1} D_{L} \\
P_{2} D_{A} & P_{2} D_{B} & -P_{2} D_{L}
\end{array}\right]^{T} \\
& +\varepsilon_{2}^{-1}\left[\begin{array}{cc}
E_{A} & E_{A} \\
E_{B} K & 0 \\
0 & E_{L} C
\end{array}\right]^{T}\left[\begin{array}{cc}
E_{A} & E_{A} \\
E_{B} K & 0 \\
0 & E_{L} C
\end{array}\right] \text {. }
\end{aligned}
$$

Thus, if the following condition holds:

$$
\begin{aligned}
& +\varepsilon_{2}\left[\begin{array}{ccc}
0 & 0 & P_{1} D_{L} \\
P_{2} D_{A} & P_{2} D_{B} & -P_{2} D_{L}
\end{array}\right]\left[\begin{array}{ccc}
0 & 0 & P_{1} D_{L} \\
P_{2} D_{A} & P_{2} D_{B} & -P_{2} D_{L}
\end{array}\right]^{T} \\
& \operatorname{Sym}\left\{\left[\begin{array}{cc}
P_{1}(\widehat{A}+B K) & P_{1} L C \\
P_{2}(A-\widehat{A}) & P_{2}(A-L C)
\end{array}\right]\right\} \quad+\varepsilon_{2}^{-1}\left[\begin{array}{cc}
E_{A} & E_{A} \\
E_{B} K & 0 \\
0 & E_{L} C
\end{array}\right]^{T}\left[\begin{array}{cc}
E_{A} & E_{A} \\
E_{B} K & 0 \\
0 & E_{L} \mathrm{C}
\end{array}\right]
\end{aligned}
$$




$$
+\varepsilon_{1}\left[\begin{array}{ll}
P_{1} & \\
& P_{2}
\end{array}\right]\left[\begin{array}{ll}
P_{1} & \\
& P_{2}
\end{array}\right]+\varepsilon_{1}^{-1} \gamma^{2} I<0
$$

then (32) will be satisfied.

Before and after multiplying both sides of (35) by the matrix $\operatorname{diag}\left\{P_{1}^{-1}, P_{2}^{-1}\right\}$ and transpose, respectively, putting $\mathscr{P}_{1}=P_{1}^{-1}, \mathscr{P}_{2}=P_{2}^{-1}$, we obtain

$$
\begin{aligned}
& \operatorname{Sym}\left\{\left[\begin{array}{cc}
(\widehat{A}+B K) \mathscr{P}_{1} & L C \mathscr{P}_{2} \\
(A-\widehat{A}) \mathscr{P}_{1} & (A-L C) \mathscr{P}_{2}
\end{array}\right]\right\} \\
& +\varepsilon_{2}\left[\begin{array}{ccc}
0 & 0 & D_{L} \\
D_{A} & D_{B} & -D_{L}
\end{array}\right]\left[\begin{array}{ccc}
0 & 0 & D_{L} \\
D_{A} & D_{B} & -D_{L}
\end{array}\right]^{T} \\
& +\varepsilon_{2}^{-1}\left[\begin{array}{cc}
E_{A} \mathscr{P}_{1} & E_{A} \mathscr{P}_{2} \\
E_{B} K \mathscr{P}_{1} & 0 \\
0 & E_{L} C \mathscr{P}_{2}
\end{array}\right]^{T}\left[\begin{array}{cc}
E_{A} \mathscr{P}_{1} & E_{A} \mathscr{P}_{2} \\
E_{B} K \mathscr{P}_{1} & 0 \\
0 & E_{L} C \mathscr{P}_{2}
\end{array}\right] \\
& +\varepsilon_{1} I+\varepsilon_{1}^{-1} \gamma^{2}\left[\begin{array}{lll}
\mathscr{P}_{1} & \\
& & \\
& \mathscr{P}_{2}
\end{array}\right]\left[\begin{array}{lll}
\mathscr{P}_{1} & \\
& & \\
& \mathscr{P}_{2}
\end{array}\right] \\
& =\operatorname{Sym}\left\{\left[\begin{array}{cc}
(\widehat{A}+B K) \mathscr{P}_{1} & L C \mathscr{P}_{2} \\
(A-\widehat{A}) \mathscr{P}_{1} & (A-L C) \mathscr{P}_{2}
\end{array}\right]\right\} \\
& +\varepsilon_{2}\left[\begin{array}{cc}
D_{L} D_{L}^{T} & -D_{L} D_{L}^{T} \\
-D_{L} D_{L}^{T} & D_{A} D_{A}^{T}+D_{B} D_{B}^{T}+D_{L} D_{L}^{T}
\end{array}\right]
\end{aligned}
$$

$$
\begin{aligned}
& +\varepsilon_{2}^{-1}\left[\begin{array}{cc}
E_{A} \mathscr{P}_{1} & E_{A} \mathscr{P}_{2} \\
E_{B} K \mathscr{P}_{1} & 0 \\
0 & E_{L} C \mathscr{P}_{2}
\end{array}\right]^{T}\left[\begin{array}{cc}
E_{A} \mathscr{P}_{1} & E_{A} \mathscr{P}_{2} \\
E_{B} K \mathscr{P}_{1} & 0 \\
0 & E_{L} C \mathscr{P}_{2}
\end{array}\right] \\
& +\varepsilon_{1} I+\varepsilon_{1}^{-1} \gamma^{2}\left[\begin{array}{cc}
\mathscr{P}_{1} & \\
& \mathscr{P}_{2}
\end{array}\right]\left[\begin{array}{cc}
\mathscr{P}_{1} & \\
& \mathscr{P}_{2}
\end{array}\right]<0 .
\end{aligned}
$$

Using Schur complement two times on (36) yields

$$
\left[\begin{array}{ccccccc}
\Omega_{11} & \Omega_{12} & \mathscr{P}_{1} & 0 & \mathscr{P}_{1} E_{A}^{T} & \mathscr{P}_{1} K^{T} E_{B}^{T} & 0 \\
* & \Omega_{22} & 0 & \mathscr{P}_{2} & \mathscr{P}_{2} E_{A}^{T} & 0 & \mathscr{P}_{2} C^{T} E_{L}^{T} \\
* & * & -\frac{\varepsilon_{1}}{\gamma^{2}} I & 0 & 0 & 0 & 0 \\
* & * & * & -\frac{\varepsilon_{1}}{\gamma^{2}} I & 0 & 0 & 0 \\
* & * & * & * & -\varepsilon_{2} I & 0 & 0 \\
* & * & * & * & * & -\varepsilon_{2} I & 0 \\
* & * & * & * & * & * & -\varepsilon_{2} I
\end{array}\right]
$$

where

$$
\begin{aligned}
\Omega_{11}= & \operatorname{Sym}\left\{(\widehat{A}+B K) \mathscr{P}_{1}\right\}+\varepsilon_{1} I+\varepsilon_{2} D_{L} D_{L}^{T}, \\
\Omega_{12}= & \mathscr{P}_{1}(A-\widehat{A})^{T}+L C \mathscr{P}_{2}-\varepsilon_{2} D_{L} D_{L}^{T}, \\
\Omega_{22}= & \operatorname{Sym}\left\{(A-L C) \mathscr{P}_{2}\right\}+\varepsilon_{1} I \\
& +\varepsilon_{2}\left(D_{A} D_{A}^{T}+D_{B} D_{B}^{T}+D_{L} D_{L}^{T}\right) .
\end{aligned}
$$

The following inequality is equivalent to (37):

$$
\left(\begin{array}{c}
z_{1}(t) \\
z_{2}(t) \\
z_{3}(t) \\
z_{4}(t) \\
z_{5}(t) \\
z_{6}(t) \\
z_{7}(t)
\end{array}\right)\left[\begin{array}{ccccccc}
\Omega_{11} & \Omega_{12} & \mathscr{P}_{1} & 0 & \mathscr{P}_{1} E_{A}^{T} & \mathscr{P}_{1} K^{T} E_{B}^{T} & 0 \\
* & \Omega_{22} & 0 & \mathscr{P}_{2} & \mathscr{P}_{2} E_{A}^{T} & 0 & \mathscr{P}_{2} C^{T} E_{L}^{T} \\
* & * & -\frac{\varepsilon_{1}}{\gamma^{2}} I & 0 & 0 & 0 & 0 \\
* & * & * & -\frac{\varepsilon_{1}}{\gamma^{2}} I & 0 & 0 & 0 \\
* & * & * & * & -\varepsilon_{2} I & 0 & 0 \\
* & * & * & * & * & -\varepsilon_{2} I & 0 \\
* & * & * & * & * & * & -\varepsilon_{2} I
\end{array}\right]\left(\begin{array}{c}
z_{1}(t) \\
z_{2}(t) \\
z_{3}(t) \\
z_{4}(t) \\
z_{5}(t) \\
z_{6}(t) \\
z_{7}(t)
\end{array}\right)<0
$$


where $z_{i}(t)(i=1,2, \ldots, 7)$ are any nonzero vectors. Now define the following new auxiliary variables:

$$
\xi(t)=C^{T} L^{T}\left(z_{1}(t)-z_{2}(t)\right) .
$$

Thus, inequality (39) can be rewritten as

$$
\left(\begin{array}{c}
z_{1}(t) \\
z_{2}(t) \\
z_{3}(t) \\
z_{4}(t) \\
z_{5}(t) \\
z_{6}(t) \\
z_{7}(t) \\
\xi(t)
\end{array}\right)\left[\begin{array}{cccccccc}
\Pi_{11} & \Pi_{12} & \mathscr{P}_{1} & 0 & \mathscr{P}_{1} E_{A}^{T} & \mathscr{P}_{1} K^{T} E_{B}^{T} & 0 & 0 \\
* & \Pi_{22} & 0 & \mathscr{P}_{2} & \mathscr{P}_{2} E_{A}^{T} & 0 & \mathscr{P}_{2} C^{T} E_{L}^{T} & \mathscr{P}_{2} \\
* & * & -\frac{\varepsilon_{1}}{\gamma^{2}} I & 0 & 0 & 0 & 0 & 0 \\
* & * & * & -\frac{\varepsilon_{1}}{\gamma^{2}} & 0 & 0 & 0 & 0 \\
* & * & * & * & -\varepsilon_{2} I & 0 & 0 & 0 \\
* & * & * & * & * & -\varepsilon_{2} I & 0 & 0 \\
* & * & * & * & * & * & -\varepsilon_{2} I & 0 \\
* & * & * & * & * & * & 0 & 0
\end{array}\right]\left(\begin{array}{c}
z_{1}(t) \\
z_{2}(t) \\
z_{3}(t) \\
z_{4}(t) \\
z_{5}(t) \\
z_{6}(t) \\
z_{7}(t) \\
\xi(t)
\end{array}\right)<0
$$

subject to

$$
\left[\begin{array}{lllllllll}
C^{T} L^{T} & -C^{T} L^{T} & 0 & 0 & 0 & 0 & 0 & -I
\end{array}\right]\left(\begin{array}{c}
z_{1}(t) \\
z_{2}(t) \\
z_{3}(t) \\
z_{4}(t) \\
z_{5}(t) \\
z_{6}(t) \\
z_{7}(t) \\
\xi(t)
\end{array}\right)=0
$$

where

$$
\begin{aligned}
\Pi_{11}= & \Omega_{11}, \\
\Pi_{12}= & \mathscr{P}_{1}(A-\widehat{A})^{T}-\varepsilon_{2} D_{L} D_{L}^{T}, \\
\Pi_{22}= & \operatorname{Sym}\left\{A \mathscr{P}_{2}\right\}+\varepsilon_{1} I \\
& +\varepsilon_{2}\left(D_{A} D_{A}^{T}+D_{B} D_{B}^{T}+D_{L} D_{L}^{T}\right) .
\end{aligned}
$$

Using Lemma 8, equality (42) holds if there exist matrix $Q \in$ $\mathbb{R}^{n \times n}$ and positive scalar $\lambda$ such that

$$
\left[\begin{array}{cccccccc}
\Pi_{11} & \Pi_{12} & \mathscr{P}_{1} & 0 & \mathscr{P}_{1} E_{A}^{T} & \mathscr{P}_{1} K^{T} E_{B}^{T} & 0 & 0 \\
* & \Pi_{22} & 0 & \mathscr{P}_{2} & \mathscr{P}_{2} E_{A}^{T} & 0 & \mathscr{P}_{2} C^{T} E_{L}^{T} & \mathscr{P}_{2} \\
* & * & -\frac{\varepsilon_{1}}{\gamma^{2}} I & 0 & 0 & 0 & 0 & 0 \\
* & * & * & -\frac{\varepsilon_{1}}{\gamma^{2}} I & 0 & 0 & 0 & 0 \\
* & * & * & * & -\varepsilon_{2} I & 0 & 0 & 0 \\
* & * & * & * & * & -\varepsilon_{2} I & 0 & 0 \\
* & * & * & * & * & * & -\varepsilon_{2} I & 0 \\
* & * & * & * & * & * & 0 & 0
\end{array}\right]
$$

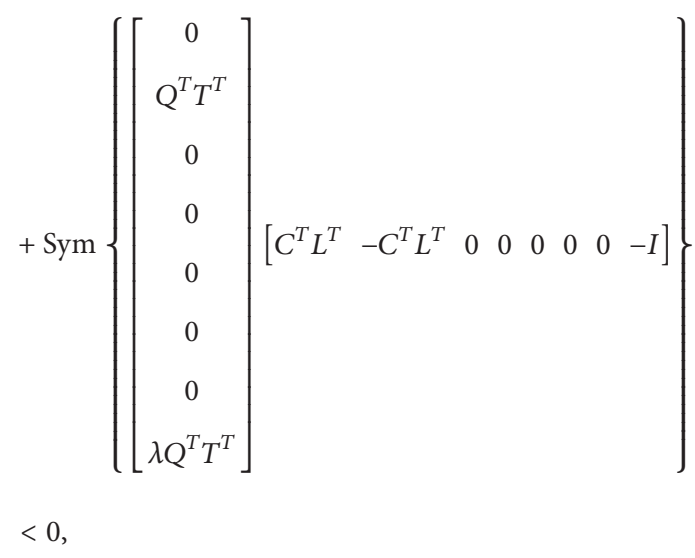

where $T$ satisfy (12). Using condition (12) in inequality (44) yields

$$
L C T Q=L\left[\begin{array}{ll}
I & 0
\end{array}\right] Q=\left[\begin{array}{ll}
L & 0
\end{array}\right] Q .
$$

Partitioning matrices $Q$ as (23), one can write (45) as follows:

$$
L C T Q=\left[\begin{array}{ll}
L Q_{11} & 0
\end{array}\right]
$$

Defining $\mathscr{L}=L Q_{11}, \widehat{Q}=[\mathscr{L} 0]$, and $\mathscr{A}=\widehat{A} \mathscr{P}_{1}, \mathscr{K}=K \mathscr{P}_{1}$, inequality (44) is converted to LMI (21). This completes the proof.

In particular, simply setting $\widehat{A}=A$ in observer (14) for nonlinear FOS (6), we have the following classical observerbased controller:

$$
\begin{aligned}
D^{\alpha} \hat{x}(t)= & A \hat{x}(t)+B u(t)+\Phi(\widehat{x}(t)) \\
& +(L+\Delta L(t))(y(t)-\widehat{y}(t)),
\end{aligned}
$$




$$
\begin{aligned}
& \widehat{y}(t)=C \widehat{x}(t), \\
& u(t)=K \widehat{x}(t) .
\end{aligned}
$$

Moreover, the stabilizing controller gain matrices $K, L$ can be solved by the following Corollary.
Corollary 14. Given positive scalar design parameter $\lambda$, nonlinear FOS (6) under the observer-based controller (47) is robustly asymptotically stable if there exist symmetric positive definite matrices $\mathscr{P}_{1}, \mathscr{P}_{2} \in \mathbb{R}^{n \times n}$, full rank matrix $Q_{11} \in$ $\mathbb{R}^{m \times m}$, matrices $Q_{21} \in \mathbb{R}^{(n-m) \times m}, Q_{22} \in \mathbb{R}^{(n-m) \times(n-m)}, \mathscr{K} \in$ $\mathbb{R}^{q \times n}, \mathscr{L} \in \mathbb{R}^{n \times m}$, and two real scalars $\varepsilon_{1}>0, \varepsilon_{2}>0$ such that the following LMI condition holds:

$$
\left[\begin{array}{cccccccc}
\widetilde{\Theta}_{11} & \widetilde{\Theta}_{12} & \mathscr{P}_{1} & 0 & \mathscr{P}_{1} E_{A}^{T} & \mathscr{K}^{T} E_{B}^{T} & 0 & \lambda \widehat{Q} \\
* & \widetilde{\Theta}_{22} & 0 & \mathscr{P}_{2} & \mathscr{P}_{2} E_{A}^{T} & 0 & \mathscr{P}_{2} C^{T} E_{L}^{T} & \mathscr{P}_{2}-Q^{T} T^{T}-\lambda \widehat{Q} \\
* & * & -\frac{\varepsilon_{1}}{\gamma^{2}} I & 0 & 0 & 0 & 0 & 0 \\
* & * & * & -\frac{\varepsilon_{1}}{\gamma^{2}} & 0 & 0 & 0 & 0 \\
* & * & * & * & -\varepsilon_{2} I & 0 & 0 & 0 \\
* & * & * & * & * & -\varepsilon_{2} I & 0 & 0 \\
* & * & * & * & * & * & -\varepsilon_{2} I & 0 \\
* & * & * & * & * & * & 0 & -\lambda\left(T Q+Q^{T} T^{T}\right)
\end{array}\right]<0 \text {, }
$$

where

$$
\begin{aligned}
& \widetilde{\Theta}_{11}= \operatorname{Sym}\left\{A \mathscr{P}_{1}+B \mathscr{K}\right\}+\varepsilon_{1} I+\varepsilon_{2} D_{L} D_{L}^{T}, \\
& \widetilde{\Theta}_{12}=\widehat{Q}-\varepsilon_{2} D_{L} D_{L}^{T}, \\
& \widetilde{\Theta}_{22}=\operatorname{Sym}\left\{A \mathscr{P}_{2}-\widehat{Q}\right\}+\varepsilon_{1} I \\
&+\varepsilon_{2}\left(D_{A} D_{A}^{T}+D_{B} D_{B}^{T}+D_{L} D_{L}^{T}\right), \\
& Q= {\left[\begin{array}{ll}
Q_{11} & 0 \\
Q_{21} & Q_{22}
\end{array}\right] \in \mathbb{R}^{n \times n}, } \\
& \widehat{Q}= {\left[\begin{array}{ll}
\mathscr{L} & 0
\end{array}\right] \in \mathbb{R}^{n \times n}, }
\end{aligned}
$$

with matrix $T$ satisfying (12). Moreover, the stabilizing control feedback and the observer gains in (47) are given by

$$
\begin{aligned}
K & =\mathscr{K} \mathscr{P}_{1}^{-1}, \\
L & =\mathscr{L} Q_{11}^{-1} .
\end{aligned}
$$

Remark 15. If $m=n$, then the expression of $Q$ and $\widehat{Q}$ can be simplified as $Q=Q_{11} \in \mathbb{R}^{n \times n}, \widehat{Q}=\mathscr{L} \in \mathbb{R}^{n \times n}$ in Theorem 13 and Corollary 14.

Remark 16. Currently, though the conditions of asymptotic stability for fractional-order system with constant timedelays have been established from the view of eigenvalues, the study of fractional-order system with time-varying delays is very difficult due to the complexity of fractional-order derivative, so, compared with the integer-order system [55, 56], it is impossible to extend the results to the FOS with timedelays until now.

\section{Simulation Examples}

In this section, to show the effectiveness of the proposed scheme, two numerical examples are presented.

Example 1. Based on the integral-order control model of highly simplified engine idle speed $[39,57]$, we consider the nonlinear FOS with fractional-order $\alpha=0.80$ represented by the following form:

$$
\begin{aligned}
D^{\alpha} x(t)= & (A+\Delta A(t)) x(t)+(B+\Delta B(t)) u(t) \\
& +\Phi(x(t)), \\
y(t)= & C x(t),
\end{aligned}
$$

where

$$
\begin{aligned}
A & =\left[\begin{array}{cc}
6.0225 & 0 \\
-0.4601 & -4.4420
\end{array}\right], \\
B & =\left[\begin{array}{cc}
0 & 2 \\
110 & 0
\end{array}\right], \\
C & =\left[\begin{array}{ll}
1 & 0
\end{array}\right], \\
\Phi(x(t)) & =\left[\begin{array}{cc}
38.9 x_{2}^{2}(t) & 0
\end{array}\right], \\
D_{A} & =\left[\begin{array}{ll}
0.5 & 0.5 \\
0.5 & 0.5
\end{array}\right], \\
E_{A} & =\left[\begin{array}{cc}
-0.3559 & 0 \\
0.3140 & -0.4038
\end{array}\right],
\end{aligned}
$$




$$
\begin{aligned}
F_{A}(t) & =\left[\begin{array}{cc}
\sin (0.2 \pi t) & 0 \\
0 & \sin (0.2 \pi t)
\end{array}\right], \\
D_{B} & =\left[\begin{array}{cc}
0 & 2.9656 \\
1 & 0
\end{array}\right], \\
E_{B} & =\left[\begin{array}{cc}
0 & 0.2 \\
2.5 & 0
\end{array}\right], \\
F_{B}(t) & =\left[\begin{array}{cc}
0 & \sin (0.2 \pi t) \\
\sin (0.2 \pi t) & 0
\end{array}\right] .
\end{aligned}
$$

Now, under the nonfragile observer-based controller (14) and (15), we consider the asymptotical stabilization of nonlinear FOS (51) with

$$
\begin{aligned}
D_{L} & =\left[\begin{array}{ll}
2 & 0 \\
0 & 2
\end{array}\right], \\
E_{L} & =\left[\begin{array}{l}
1 \\
1
\end{array}\right], \\
F_{L}(t) & =\left[\begin{array}{cc}
0 & \sin (0.1 \pi t) \\
\cos (0.1 \pi t) & 0
\end{array}\right] .
\end{aligned}
$$

Let $\gamma=2, \lambda=0.01$; based on Theorem 13, LMI condition (21) can be solved by Matlab's LMI toolbox; the solution of LMI (21) is presented as follows:

$$
\begin{aligned}
\mathscr{P}_{1} & =\left[\begin{array}{ll}
0.7750 & 0.0293 \\
0.0293 & 1.3870
\end{array}\right], \\
\mathscr{P}_{2} & =\left[\begin{array}{ll}
1.0455 & 0.0100 \\
0.0100 & 3.4553
\end{array}\right], \\
Q_{11} & =\left[\begin{array}{ll}
0.8517
\end{array}\right], \\
\mathscr{A} & =\left[\begin{array}{ll}
22.3690 & 0.6336 \\
-0.5263 & -15.5467
\end{array}\right], \\
\mathscr{K} & =\left[\begin{array}{cc}
-0.0017 & 0.0369 \\
-19.2764 & 0.0013
\end{array}\right], \\
\mathscr{L} & =\left[\begin{array}{ll}
29.0789 \\
0.5615
\end{array}\right], \\
\mathcal{E}_{1} & =8.8803 \\
\mathcal{E}_{2} & =2.4249 .
\end{aligned}
$$

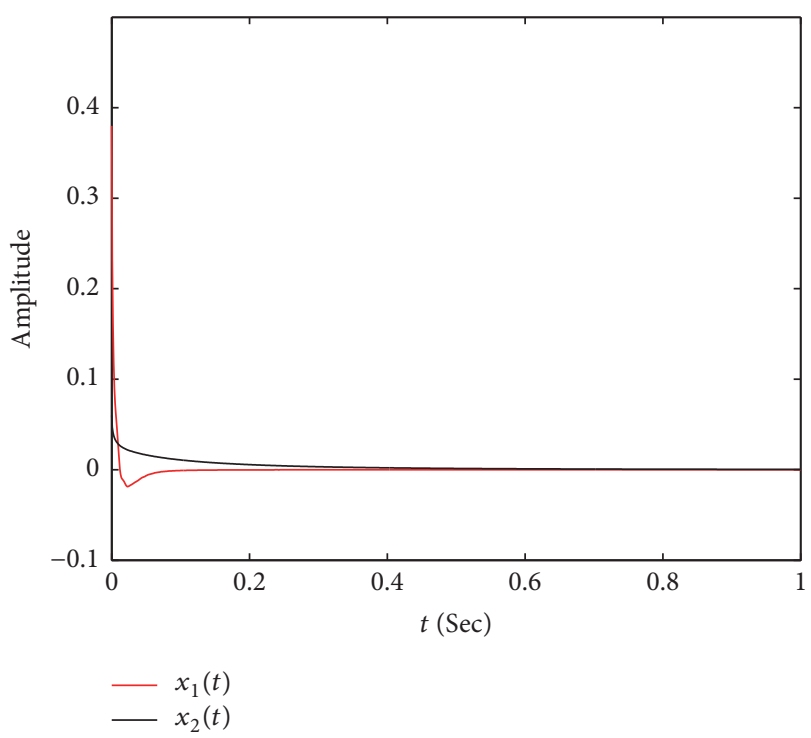

FIgure 1: The state of fractional-order system (51).

Thus, the feedback gain $K$ and the observer gains $\widehat{A}, L$ in (14) and (15) are given by

$$
\begin{aligned}
& \widehat{A}=\mathscr{A} \mathscr{P}_{1}^{-1}=\left[\begin{array}{cc}
28.8680 & -0.1533 \\
-0.2554 & -11.2031
\end{array}\right], \\
& K=\mathscr{K} \mathscr{P}_{1}^{-1}=\left[\begin{array}{cc}
-0.0032 & 0.0266 \\
-24.8919 & 0.5270
\end{array}\right], \\
& L=\mathscr{L} Q_{11}^{-1}=\left[\begin{array}{c}
34.1437 \\
0.6593
\end{array}\right] .
\end{aligned}
$$

Finally, by a similar procedure in reference [39], we use a recursive poles/zeros filter of order four to approximate the fractional operator $1 / s^{0.8}$, and we can obtain the following initial conditions: $x_{0}=(0.38,0.06)^{T}, e_{0}=(0,0)^{T}$; the time response of state, state estimate, and observation errors for system (51) based on the nonfragile observer (14) with state estimated feedback control law (15) are shown in Figures 1, 2 , and 3, respectively. From these three figures, it is clear that the closed-loop system is asymptotically stable and the observation errors also tend to zeros.

Example 2. We apply them in observer design a fractionalorder chaotic Lorenz system with fractional-order $\alpha=0.995$. The fractional-order Lorenz system [38] can be expressed as

$$
\begin{aligned}
D^{\alpha} x(t) & =A x(t)+\Phi(x(t)), \\
y(t) & =C x(t),
\end{aligned}
$$




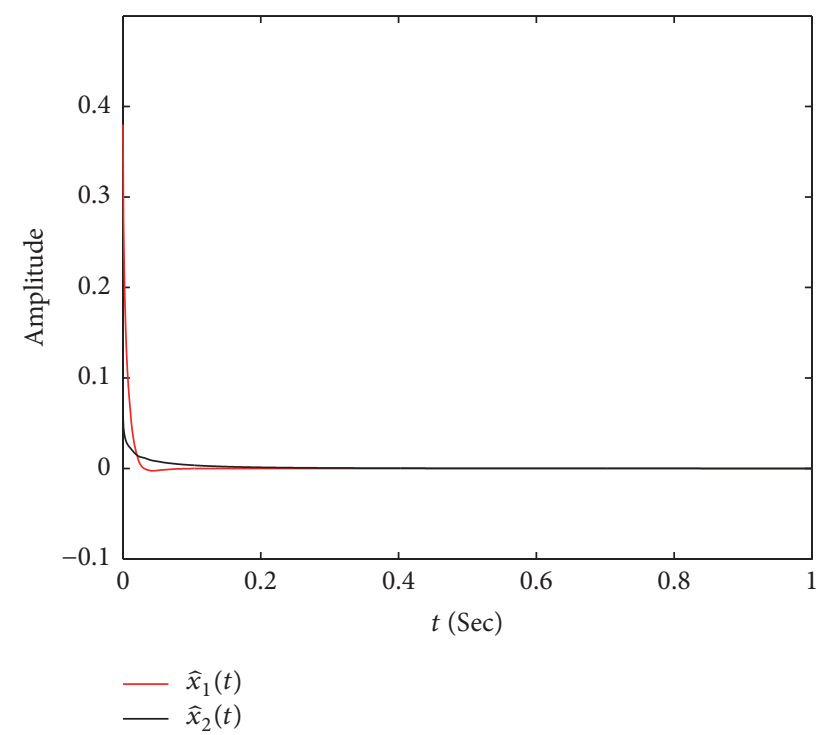

FIGURE 2: The state estimate of fractional-order system (51).

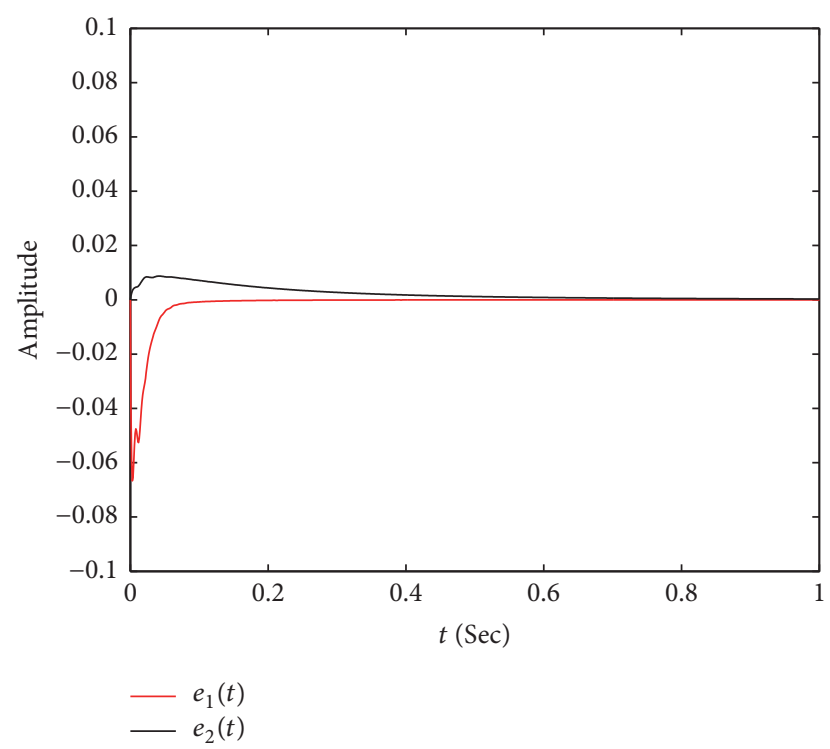

FIGURE 3: The observation errors of fractional-order system (51).

where

$$
\begin{aligned}
A & =\left[\begin{array}{ccc}
-10 & 10 & 0 \\
28 & -1 & 0 \\
0 & 0 & -\frac{8}{3}
\end{array}\right], \\
\Phi(x(t)) & =\left[\begin{array}{c}
0 \\
-x_{1}(t) x_{3}(t) \\
x_{1}(t) x_{2}(t)
\end{array}\right], \\
C & =\left[\begin{array}{lll}
1 & 0 & 0
\end{array}\right],
\end{aligned}
$$

and $x=\left(x_{1}, x_{2}, x_{3}\right)^{T}$. The fractional-order Lorenz system (56) has a chaotic attractor as depicted in Figure 4.

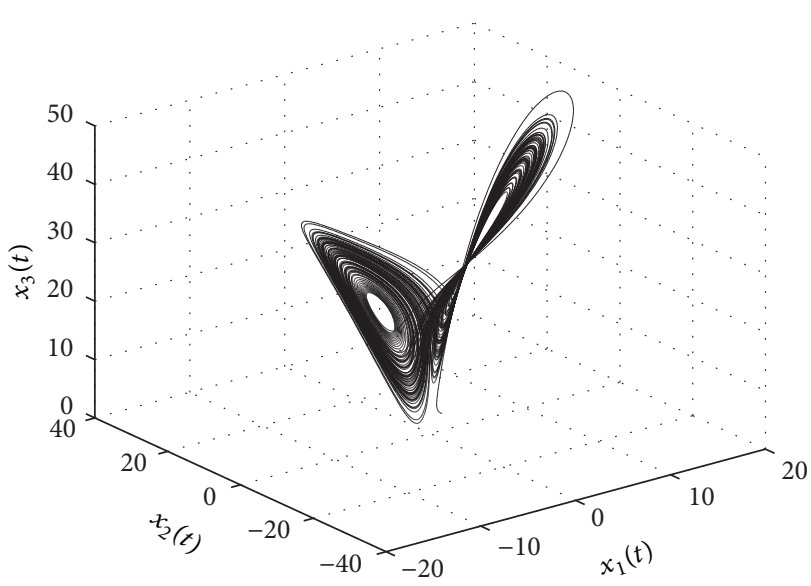

FIGURE 4: Chaotic behaviors of fractional-order chaotic Lorenz system (56).

Obviously, it is easy to verify that

$$
\begin{aligned}
\|\Phi(x(t))\| & =\sqrt{\left(x_{1} x_{3}\right)^{2}+\left(x_{1} x_{2}\right)^{2}} \\
& \leq\left\|x_{1}\right\| \sqrt{x_{1}^{2}+x_{2}^{2}+x_{3}^{2}} \leq \gamma\|x\|,
\end{aligned}
$$

which implies that $\Phi(x(t))$ satisfies Lipschitz-conditions (9). For simplicity, select $B=\left[\begin{array}{lll}2 & 7 & 3\end{array}\right]^{T}$ and add the uncertainties as follows:

$$
\begin{aligned}
& D_{A}=\left[\begin{array}{ccc}
-0.5 & 0.5 & 0.1 \\
0.1 & -0.5 & 0.2 \\
0.2 & 0.2 & -0.1
\end{array}\right], \\
& E_{A}=\left[\begin{array}{lll}
0.3 & 0.1 & 0.1 \\
0.3 & 0.4 & 0.1 \\
0.1 & 0.2 & 0.1
\end{array}\right],
\end{aligned}
$$$$
D_{B}=\left[\begin{array}{ccc}
0 & 0.2 & 0 \\
0.2 & 0.1 & 0 \\
0.2 & 0.5 & 0.1
\end{array}\right],
$$

$$
\begin{aligned}
E_{B} & =\left[\begin{array}{l}
0.2 \\
0.5 \\
0.1
\end{array}\right], \\
F_{A}(t) & =\left[\begin{array}{ccc}
\sin (0.1 \pi t) & 0 & \\
0 & \cos (0.2 \pi t) & 0 \\
0 & 0 & \cos (0.2 \pi t)
\end{array}\right], \\
F_{B}(t) & =\left[\begin{array}{ccc}
\sin (0.1 \pi t) & 0 & \\
0 & \cos (0.2 \pi t) & 0 \\
0 & 0 & \cos (0.2 \pi t)
\end{array}\right] .
\end{aligned}
$$


Now, under the nonfragile observer-based controller (14) and (15), we consider the asymptotical stabilization problem of nonlinear FOS (56) with

$$
\begin{aligned}
D_{L} & =\left[\begin{array}{ll}
0.2 & 0.1 \\
0.2 & 0.2 \\
0.5 & 0.1
\end{array}\right], \\
E_{L} & =\left[\begin{array}{l}
0.1 \\
0.1
\end{array}\right], \\
F_{L}(t) & =\left[\begin{array}{cc}
\sin (0.1 \pi t) & \cos (0.2 \pi t)
\end{array}\right] .
\end{aligned}
$$

Let $\gamma=2, \lambda=0.01$; based on Theorem 13, LMI condition (21) can be solved by Matlab's LMI toolbox; the solution of LMI (21) is presented as follows:

$$
\begin{aligned}
\mathscr{P}_{1} & =\left[\begin{array}{lll}
2.0589 & 1.0382 & 1.6892 \\
1.0382 & 7.6562 & 8.4650 \\
1.6892 & 8.4650 & 24.8638
\end{array}\right], \\
\mathscr{P}_{2} & =\left[\begin{array}{ccc}
4.7320 & -1.8797 & -0.1000 \\
-1.8797 & 3.6335 & 0.2624 \\
-0.1000 & 0.2624 & 29.5761
\end{array}\right], \\
Q_{11} & =\left[\begin{array}{lll}
3.8079
\end{array}\right], \\
\mathscr{A} & =\left[\begin{array}{ccc}
1.6444 & 206.0205 & 83.0534 \\
47.9211 & 27.1592 & 34.9467 \\
-2.5506 & -23.2978 & -76.6106
\end{array}\right], \\
\mathscr{L} & =\left[\begin{array}{c}
4.5843 \\
169.4311 \\
13.1177
\end{array}\right], \\
\mathscr{K} & =[-32.3753-14.83951 .4081], \\
\mathcal{E}_{1} & =75.0263, \\
\mathcal{E}_{2} & =29.2692 .
\end{aligned}
$$

Thus, the feedback gain $K$ and the observer gains $\widehat{A}, L$ in (14) and (15) are given by

$$
\begin{aligned}
\widehat{A} & =\mathscr{A} \mathscr{P}_{1}^{-1}=\left[\begin{array}{ccc}
-11.1833 & 38.3148 & -8.9443 \\
23.2023 & 0.9460 & -0.4929 \\
1.2554 & 0.4615 & -3.3236
\end{array}\right], \\
L & =\mathscr{L} Q_{11}^{-1}=\left[\begin{array}{c}
1.2039 \\
44.4950 \\
3.4449
\end{array}\right], \\
K & =\mathscr{K} \mathscr{P}_{1}^{-1}=\left[\begin{array}{lll}
-16.3145 & -1.6261 & 1.7187
\end{array}\right] .
\end{aligned}
$$

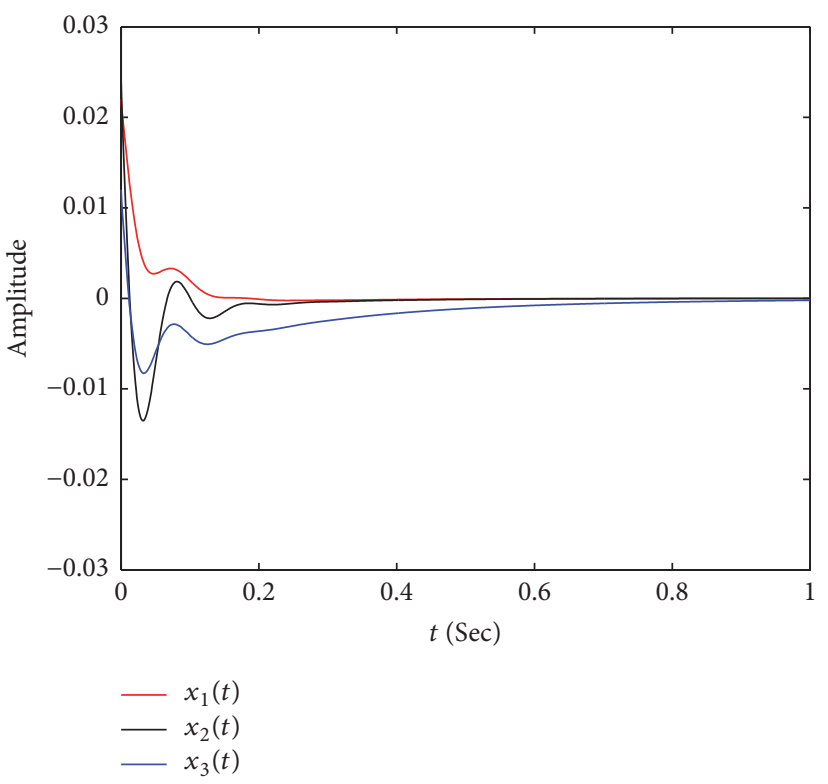

FIgURE 5: The state of fractional-order chaotic Lorenz system (56).

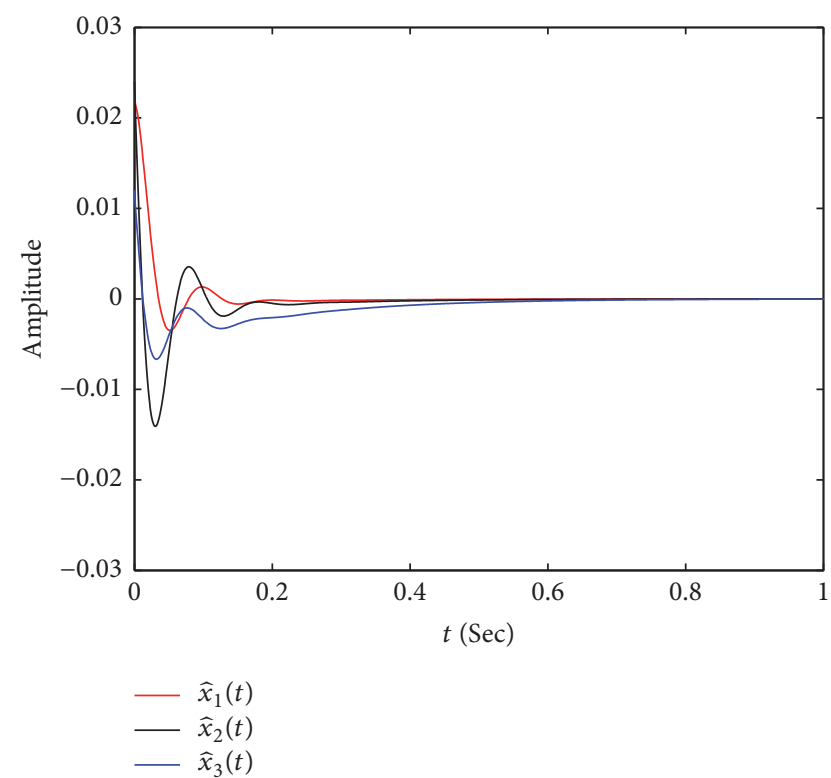

FIGURE 6: The state estimate of fractional-order chaotic Lorenz system (56).

Finally, by a similar procedure to that in reference [39], we use a recursive poles/zeros filter of order four to approximate the fractional operator $1 / s^{0.995}$, and we can obtain the following initial conditions: $x_{0}=(0.022,0.024,0.012)^{T}$, $e_{0}=(0,0,0)^{T}$; the time response of state, state estimate, and observation errors for system (56) based on the nonfragile observer (14) with state estimated feedback control law (15) are shown in Figures 5, 6, and 7, respectively. From these three figures, it is clear that the closed-loop system is asymptotically stable and the observation errors also tend to zeros. 


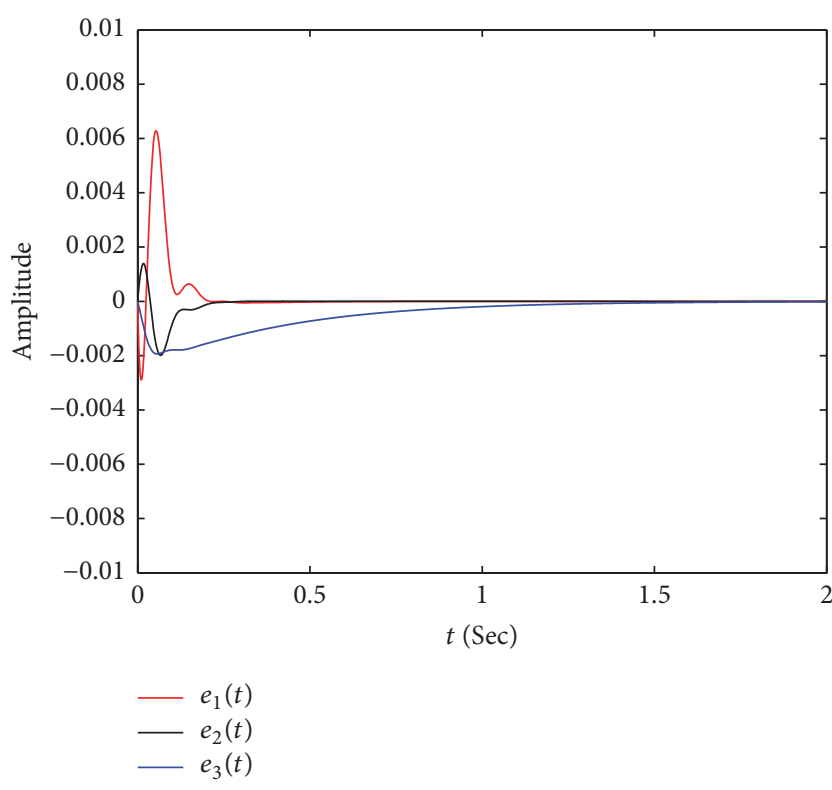

FIGURE 7: The observation errors of fractional-order chaotic Lorenz system (56).

Remark 17. For the problem of observer-based controller design, linearizing the matrix inequality is a very difficult task due to the appearance of nonlinear term. In general, by introducing a new matrix satisfying the additional matrix equation condition, the matrix inequality with nonlinear term can be transformed into the form of LMI based on the matrix's SVD. However, the matrix's SVD method is essentially conservative. In order to overcome this problem, we propose a new pattern to overcome the barrier of nonlinear term without matrix equation constraint.

\section{Conclusion}

In this work, an observer-based robust controller design method for nonlinear fractional-order uncertain systems was proposed. By employing Finsler's Lemma, the sufficient conditions for stability of closed-loop systems are derived, and therefore the gain matrices can be derived by the solvability of relevant LMIs. The resulting LMIs must be solved to obtain the feedback controller matrix as well as the observer gain matrix. The effectiveness of the design method was shown by two simulation examples.

\section{Conflicts of Interest}

The authors declare that there are no conflicts of interest regarding the publication of this paper.

\section{Acknowledgments}

This work is partially supported by the National Natural Science Foundation of China (61300120), the Natural Science
Foundation of Hebei Province (A2015208051), and the FivePlatform Foundation of Hebei University of Science and Technology (2015PT21).

\section{References}

[1] I. Podlubny, Fractional Differential Equations, vol. 198 of Mathematics in Science and Engineering, Academic Press, San Diego, Calif, USA, 1999.

[2] C. A. Monje, Y. Chen, B. M. Vinagre, D. Xue, and V. Feliu, Fractional-Order Systems and Controls, Advances in Industrial Control, Springer, London, UK, 2010.

[3] Z. Liao, C. Peng, W. Li, and Y. Wang, "Robust stability analysis for a class of fractional order systems with uncertain parameters," Journal of the Franklin Institute. Engineering and Applied Mathematics, vol. 348, no. 6, pp. 1101-1113, 2011.

[4] N. Tan, Ö. Faruk Özgüven, and M. Mine Özyetkin, "Robust stability analysis of fractional order interval polynomials," ISA Transactions, vol. 48, no. 2, pp. 166-172, 2009.

[5] H.-S. Ahn and Y. Chen, "Necessary and sufficient stability condition of fractional-order interval linear systems," Automatica, vol. 44, no. 11, pp. 2985-2988, 2008.

[6] A. Dzieliński and D. Sierociuk, "Stability of discrete fractional order state-space systems," Journal of Vibration and Control, vol. 14, no. 9-10, pp. 1543-1556, 2008.

[7] J.-G. Lu and Y.-Q. Chen, "Robust Stability and Stabilization of Fractional-Order Interval Systems with the Fractional Order $\alpha$ : The $0 \ll \alpha \ll 1$ Case," IEEE Transactions on Automatic Control, vol. 55, no. 1, pp. 152-158, 2010.

[8] M. Chilali, P. Gahinet, and P. Apkarian, "Robust pole placement in LMI regions," Institute of Electrical and Electronics Engineers. Transactions on Automatic Control, vol. 44, no. 12, pp. 22572270, 1999.

[9] Y. Li, Y. Chen, and I. Podlubny, "Stability of fractional-order nonlinear dynamic systems: Lyapunov direct method and generalized Mittag-Leffler stability," Computers and Mathematics with Applications, vol. 59, no. 5, pp. 1810-1821, 2010.

[10] J. Yu, H. Hu, S. Zhou, and X. Lin, "Generalized Mittag-Leffler stability of multi-variables fractional order nonlinear systems," Automatica, vol. 49, no. 6, pp. 1798-1803, 2013.

[11] N. Aguila-Camacho, M. A. Duarte-Mermoud, and J. A. Gallegos, "Lyapunov functions for fractional order systems," Communications in Nonlinear Science and Numerical Simulation, vol. 19, no. 9, pp. 2951-2957, 2014.

[12] D. Chen, R. Zhang, X. Liu, and X. Ma, "Fractional order Lyapunov stability theorem and its applications in synchronization of complex dynamical networks," Communications in Nonlinear Science and Numerical Simulation, vol. 19, no. 12, pp. 4105-4121, 2014.

[13] J. C. Trigeassou and N. Maamri, "Initial conditions and initialization of linear fractional differential equations," Signal Processing, vol. 91, no. 3, pp. 427-436, 2011.

[14] J. C. Trigeassou, N. Maamri, J. Sabatier, and A. Oustaloup, "A Lyapunov approach to the stability of fractional differential equations," Signal Processing, vol. 91, no. 3, pp. 437-445, 2011.

[15] I. N’Doye, M. Darouach, M. Zasadzinski, and N.-E. Radhy, "Robust stabilization of uncertain descriptor fractional-order systems," Automatica, vol. 49, no. 6, pp. 1907-1913, 2013.

[16] Y. Yu, Z. Jiao, and C.-Y. Sun, "Sufficient and necessary condition of admissibility for fractional-order singular system," Acta 
Automatica Sinica. Zidonghua Xuebao, vol. 39, no. 12, pp. 21602164, 2013.

[17] Y. Ji and J. Qiu, "Stabilization of fractional-order singular uncertain systems," ISA Transactions, vol. 56, pp. 53-64, 2015.

[18] C. Bonnet and J. R. Partington, "Analysis of fractional delay systems of retarded and neutral type," Automatica, vol. 38, no. 8, pp. 1133-1138, 2002.

[19] X. Zhang, "Some results of linear fractional order time-delay system," Applied Mathematics and Computation, vol. 197, no. 1, pp. 407-411, 2008.

[20] C. P. Li and F. R. Zhang, "A survey on the stability of fractional differential equations," European Physical Journal: Special Topics, vol. 193, no. 1, pp. 27-47, 2011.

[21] M. Rivero, S. V. Rogosin, J. A. Tenreiro Machado, and J. J. Trujillo, "Stability of fractional order systems," Mathematical Problems in Engineering, vol. 2013, Article ID 356215, 14 pages, 2013.

[22] X.-M. Zhang and Q.-L. Han, "Event-based $H_{\infty}$ filtering for sampled-data systems," Automatica, vol. 51, pp. 55-69, 2015.

[23] X.-M. Zhang and Q.-L. Han, "Network-based $H_{\infty}$ filtering using a logic jumping-like trigger," Automatica, vol. 49, no. 5, pp. 1428-1435, 2013.

[24] J. Sabatier, C. Farges, M. Merveillaut, and L. Feneteau, "On observability and pseudo state estimation of fractional order systems," European Journal of Control, vol. 18, no. 3, pp. 260271, 2012.

[25] Y.-H. Lan, H.-X. Huang, and Y. Zhou, "Observer-based robust control of a $(1 \leq \alpha<2)$ fractional-order uncertain systems: a linear matrix inequality approach," IET Control Theory \& Applications, vol. 6, no. 2, pp. 229-234, 2012.

[26] E. A. Boroujeni and H. R. Momeni, "Non-fragile nonlinear fractional order observer design for a class of nonlinear fractional order systems," Signal Processing, vol. 92, no. 10, pp. 2365-2370, 2012.

[27] J. Huang and Z. Han, "Adaptive non-fragile observer design for the uncertain Lur'e differential inclusion system," Applied Mathematical Modelling, vol. 37, no. 1-2, pp. 72-81, 2013.

[28] M. H. Asemani and V. J. Majd, "A robust $H_{\infty}$ observerbased controller design for uncertain T-S fuzzy systems with unknown premise variables via LMI," Fuzzy Sets and Systems, vol. 212, pp. 21-40, 2013.

[29] I. N’Doye, M. Darouach, H. Voos, and M. Zasadzinski, “Design of unknown input fractional-order observers for fractionalorder systems," International Journal of Applied Mathematics and Computer Science, vol. 23, no. 3, pp. 491-500, 2013.

[30] D. Xie, N. Xu, and X. Chen, "Stabilisability and observerbased switched control design for switched linear systems," IET Control Theory and Applications, vol. 2, no. 3, pp. 192-199, 2008.

[31] S. Ibrir and M. Bettayeb, "New sufficient conditions for observer-based control of fractional-order uncertain systems," Automatica, vol. 59, pp. 216-223, 2015.

[32] J. Tsinias, “Observer design for nonlinear systems," Systems and Control Letters, vol. 13, no. 2, pp. 135-142, 1989.

[33] R. Rajamani, "Observers for Lipschitz nonlinear systems," Institute of Electrical and Electronics Engineers. Transactions on Automatic Control, vol. 43, no. 3, pp. 397-401, 1998.

[34] E. D. Sontag and Y. Wang, "Output-to-state stability and detectability of nonlinear systems," Systems and Control Letters, vol. 29, no. 5, pp. 279-290, 1997.

[35] J. P. Hespanha and A. S. Morse, "Certainty equivalence implies detectability," Systems and Control Letters, vol. 36, no. 1, pp. 1-13, 1999.
[36] Y.-H. Lan, H.-B. Gu, C.-X. Chen, Y. Zhou, and Y.-P. Luo, "An indirect Lyapunov approach to the observer-based robust control for fractional-order complex dynamic networks," Neurocomputing, vol. 136, pp. 235-242, 2014.

[37] C. Li, J. Wang, J. Lu, and Y. Ge, "Observer-based stabilisation of a class of fractional order non-linear systems for $0<\alpha<$ 2 case," IET Control Theory and Applications, vol. 8, no. 13, pp. 1238-1246, 2014.

[38] I. N'Doye, H. Voos, and M. Darouach, “Observer-based approach for fractional-order chaotic synchronization and secure communication," IEEE Journal on Emerging and Selected Topics in Circuits and Systems, vol. 3, no. 3, pp. 442-450, 2013.

[39] Y.-H. Lan and Y. Zhou, "Non-fragile observer-based robust control for a class of fractional-order nonlinear systems," Systems and Control Letters, vol. 62, no. 12, pp. 1143-1150, 2013.

[40] M. Pourgholi and V. J. Majd, "A nonlinear adaptive resilient observer design for a class of Lipschitz systems using LMI," Circuits, Systems, and Signal Processing, vol. 30, no. 6, pp. 14011415, 2011.

[41] Y.-H. Lan, W.-J. Li, Y. Zhou, and Y.-P. Luo, "Non-fragile observer design for fractional-order one-sided Lipschitz nonlinear systems," International Journal of Automation and Computing, vol. 10, no. 4, pp. 296-302, 2013.

[42] M. Pourgholi and E. A. Boroujeni, "An iterative LMI-based reduced-order observer design for fractional-order chaos synchronization," Circuits, Systems, and Signal Processing, vol. 35, no. 6, pp. 1855-1870, 2016.

[43] C. Li, J. Wang, and J. Lu, “Observer-based robust stabilisation of a class of non-linear fractional-order uncertain systems: An linear matrix inequalitie approach," IET Control Theory and Applications, vol. 6, no. 18, pp. 2757-2764, 2012.

[44] N. Djeghali, S. Djennoune, M. Bettayeb, M. Ghanes, and J.-P. Barbot, "Observation and sliding mode observer for nonlinear fractional-order system with unknown input," ISA Transactions, vol. 63, pp. 1-10, 2016.

[45] J. C. Trigeassou, N. Maamri, J. Sabatier, and A. Oustaloup, "State variables and transients of fractional order differential systems," Computers \& Mathematics with Applications, vol. 64, no. 10, pp. 3117-3140, 2012.

[46] J. C. Trigeassou, N. Maamri, J. Sabatier, and A. Oustaloup, "Transients of fractional-order integrator and derivatives," Signal, Image and Video Processing, vol. 6, no. 3, pp. 359-372, 2012.

[47] J. C. Trigeassou, N. Maamri, and A. Oustaloup, "The infinite state approach: Origin and necessity," Computers and Mathematics with Applications, vol. 66, no. 5, pp. 892-907, 2013.

[48] J. Sabatier, M. Merveillaut, R. Malti, and A. Oustaloup, "How to impose physically coherent initial conditions to a fractional system?" Communications in Nonlinear Science and Numerical Simulation, vol. 15, no. 5, pp. 1318-1326, 2010.

[49] J. Sabatier and C. Farges, "Long memory models: A first solution to the infinite energy storage ability of linear time-invariant fractional models," IFAC Proceedings Volumes, vol. 47, no. 3, pp. 2884-2890, 2014.

[50] J. Sabatier and C. Farges, "Analysis of fractional models physical consistency," Journal of Vibration and Control, vol. 23, no. 6, pp. 895-908, 2017.

[51] L. Xie, "Output feedback $H_{\infty}$ control of systems with parameter uncertainty," International Journal of Control, vol. 63, no. 4, pp. 741-750, 1996.

[52] R. E. Skelton, T. Iwasaki, and K. M. Grigoriadis, A Unified Algebraic Approach to Linear Control Design, The Taylor \& 
Francis Systems and Control Book Series, Taylor \& Francis, London, UK, 1998.

[53] M. C. de Oliveira and R. E. Skelton, "Stability tests for constrained linear systems," in Perspectives in Robust Control (Newcastle, 2000), S. O. Reza Moheimani, Ed., vol. 268 of Lecture Notes in Control and Information Science, pp. 241-257, Springer, London, UK, 2001.

[54] X. Du and G.-H. Yang, "New characterisations of positive and static output feedback control of discrete-time systems," International Journal of Control, vol. 82, no. 8, pp. 1485-1495, 2009.

[55] X.-M. Zhang and Q.-L. Han, "Event-triggered $H_{\infty}$ control for a class of nonlinear networked control systems using novel integral inequalities," International Journal of Robust and Nonlinear Control, vol. 27, no. 4, pp. 679-700, 2017.

[56] X.-M. Zhang and Q.-L. Han, "Global asymptotic stability for a class of generalized neural networks with interval time-varying delays," IEEE Transactions on Neural Networks, vol. 22, no. 8, pp. 1180-1192, 2011.

[57] Y.-H. Lan and H.-X. Huang, "Observer-based robust control of time-delay uncertain systems with application to engine idle speed control," International Journal of Dynamical Systems and Differential Equations, vol. 4, no. 3, pp. 274-285, 2012. 


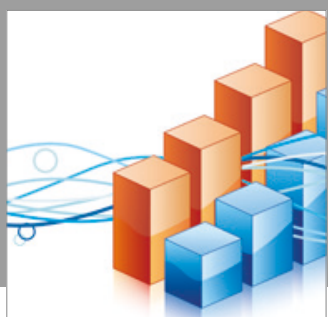

Advances in

Operations Research

vatersals

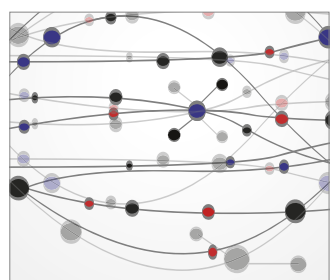

\section{The Scientific} World Journal
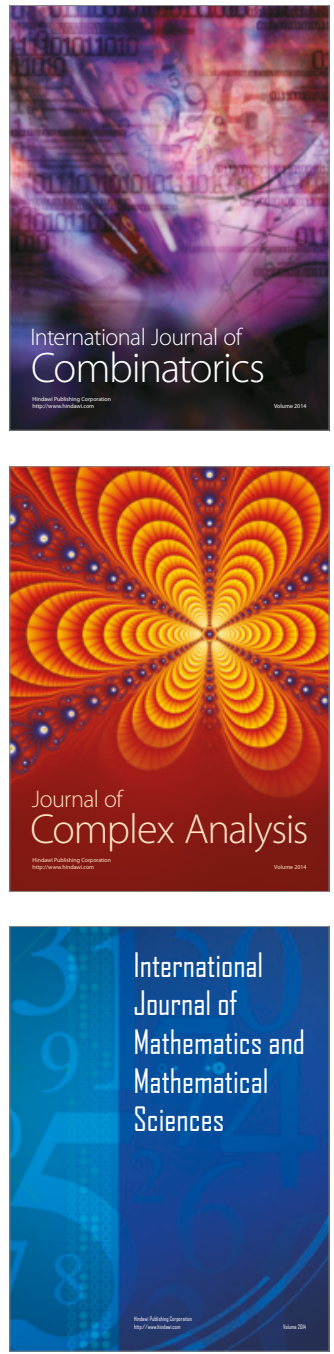
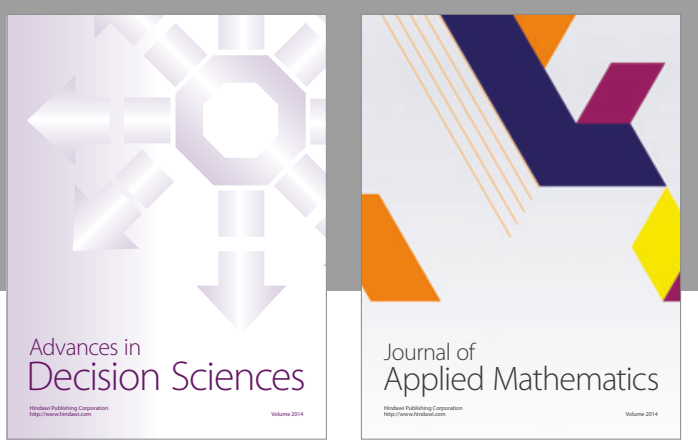

Algebra

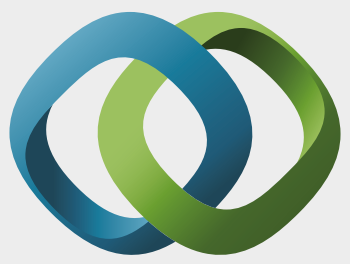

\section{Hindawi}

Submit your manuscripts at

https://www.hindawi.com
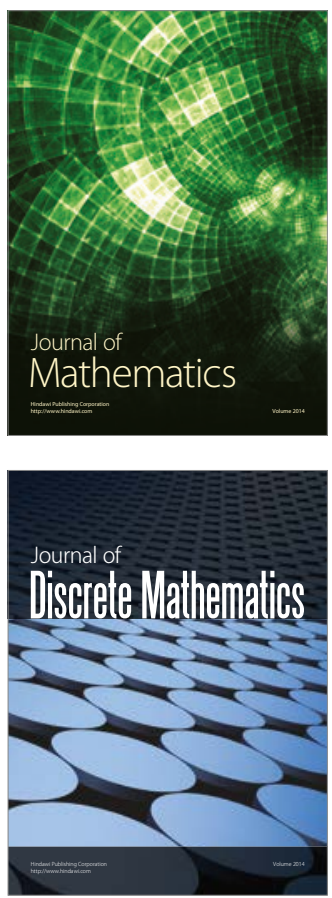

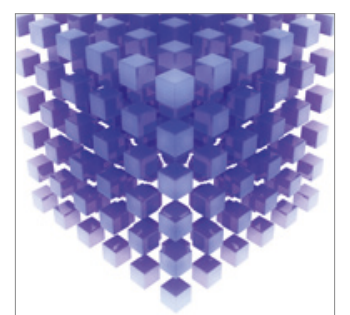

Mathematical Problems in Engineering
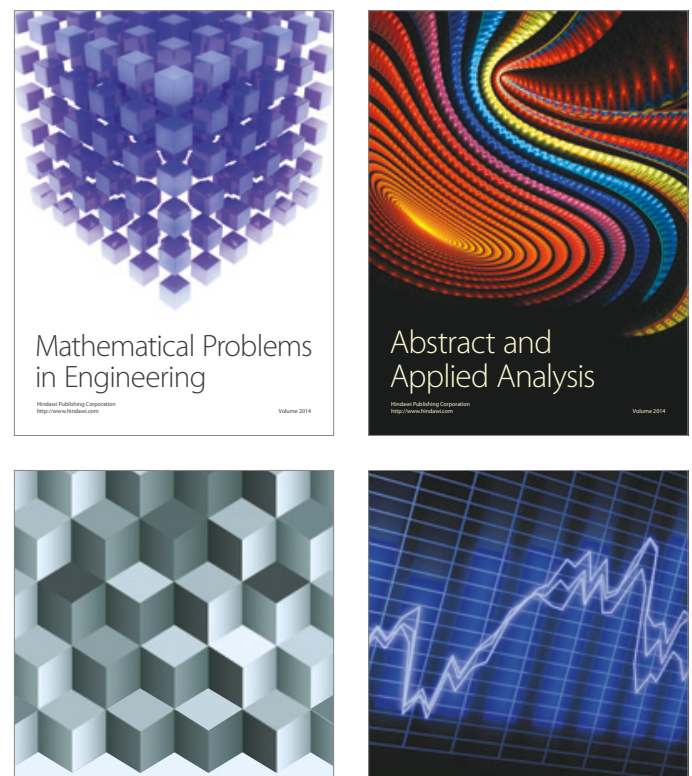

Journal of

Function Spaces

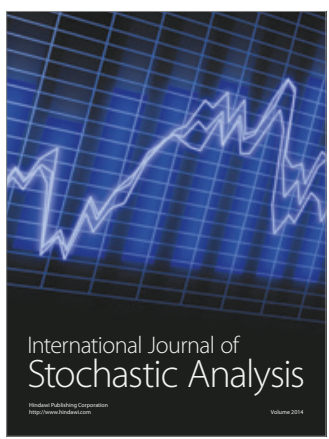

Probability and Statistics
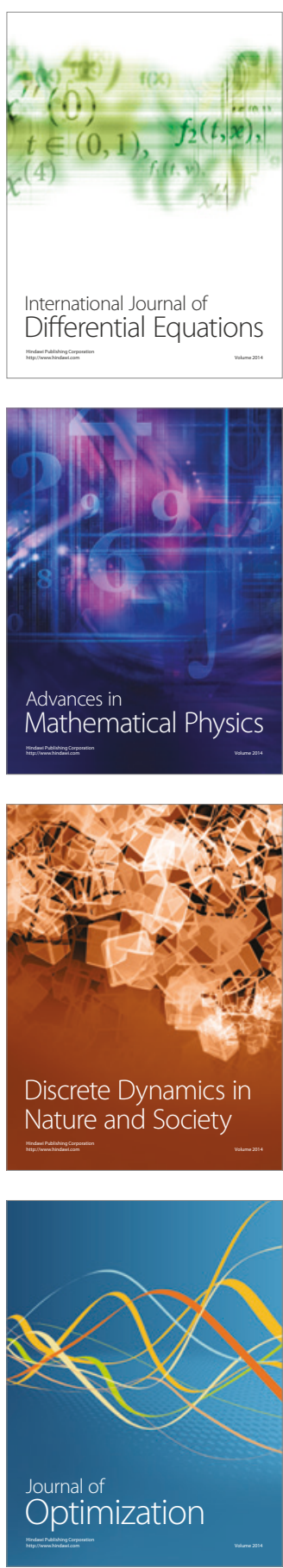